\title{
Factores clave de una central de compras cooperativa. Análisis comparativo de dos experiencias
}

\author{
Carmen Boccatonda \\ Lucía-Clara Banchieri \\ Fernando Campa-Planas
}

RESUMEN: El entorno competitivo y dinámico ha obligado a las empresas a buscar la excelencia operativa y la mayor eficiencia para sobrevivir, no estando las empresas cooperativas exentas de dicha búsqueda. Una de las herramientas de que dispone este tipo de organizaciones es unirse formando una central de compras que les permita acceder a menores costes como consecuencia del mayor volumen.

El objetivo del presente trabajo es averiguar los factores clave para el buen funcionamiento de una central de compras cooperativa y, asimismo, realizar un análisis comparativo entre la central de compras de la Federación Argentina de Cooperativas de Consumo (en adelante, CCFACC), una incipiente central de compras cooperativa argentina, y la Coop Italia, un modelo con amplia trayectoria en el mercado, con resultados comprobados y con proyecciones de mayor crecimiento.

La metodología utilizada es el estudio de caso múltiple cruzado, en donde el muestreo consiste en dos casos específicos que son escogidos por ser reveladores y ejemplos extremos. Dicho análisis presenta interés dado que, aunque cada uno de éstos se ubica en contextos y continentes diferentes, siguen compartiendo el compromiso por los valores y principios cooperativos.

PALABRAS CLAVE: Cooperativas de consumo, central de compras, estudio de casos.

CLAVES ECONLIT: P13, L81.

Cómo citar este artículo / How to cite this article: BOCCATONDA, C., BANCHIERI, L.C. \& CAMPA-PLANAS,

F. (2019): "Factores clave de una central de compras cooperativa. Análisis comparativo de dos experiencias", CIRIEC-España, Revista de Economía Pública, Social y Cooperativa, 96, 91-119. DOI: 10.7203/CIRIECE.96.10298.

Correspondencia: Carmen Boccatonda, Universidad Nacional del Sur (Argentina), carmenboccatonda@gmail.com; Lucía-Clara Banchieri, Universidad Nacional del Sur (Argentina), lucia.banchieri@uns.edu.ar, y Fernando Campa-Planas, Universitat Rovira i Virgili, Reus (Tarragona), fernando.campa@urv.cat. 


\section{EXPANDED ABSTRACT}

\section{Key factors of a cooperative central purchasing office. Comparative analysis of two experiences}

The competitive and dynamic environment has moved the companies to look for an operative excellence and the greater efficiency to survive, not being the cooperative companies out of this phenomenon. One of the tools that this type of organizations have is to create central purchasing office that should allow them to decrease their costs as consequence of the higher volume.

In this sense, the concept of "central purchasing office" is defined as a mechanism that allows to concentrate different purchase needs, with the aim to improve the competitive position of each associated company. Blond (2017) said that, "the cooperatives of second degree constitute the mechanism by excellence of cooperative integration" (p.119).

Additionally, according to the definition of "central purchasing office" of the Asociación de Centrales de Compras y Servicios de España (from now on, ANCECO), these bodies guarantee the diversity of formats, as far as they allow that the small and medium companies may compete to the same level that the big companies (ANCECO, 2016).

At the same time, in the plan for a cooperative decade, the Alianza Cooperativa Internacional (from now on, $\mathrm{ACl}$ ) (2013) exposes that the goal is the solid affirmation that the different organization coming from the cooperative is better that the one that try to maximize their profits. This vision stands out the cooperative identity, as well as the sustainability and the participation of the partners of each cooperative.

The aim of this investigation is to detect the key factors for a good management of a cooperative central purchasing office and are analyze how is managed each of these variables of selected. The comparative analysis results interesting since two extreme cases are chosen: on one hand, the Federación Argentina de Cooperativas de Consumo (from now on, CCFACC), with only a year of operation, and, in the other hand the Coop Italy, a model with tradition in the market, with results checked and with projections of greater growth. An additional richness for the analysis is that each one of the cases is in different contexts and geographical continents.

The methodology used is the study of multiple case crossed, where the sampling consists in two specific cases that are chosen for being relevant and extreme examples. Although each one of these cases are situated under different environments, both are sharing the commitment for the values and cooperative principles. 
On the other hand, to detect the key factors that contribute to the development of a central purchasing office, three studies have been analyzed, which investigate the factors of greater incidence for a successful cooperative operation. It is possible that in several cases the key factors have relation with the problems detected, which would confirm the importance of the best practices. The works reviewed were the following: Hoffmann and Schlosser (2001), Lagemann (2004) and Torres Ruiz et al. (2006).

After having detected the key factors and have made the analysis between the two units of analysis selected on how influence each one in relation to each variable, it has been verified that the two offices act proactively in relation to these and, consequently, achieve a satisfactory performance.

It has been confirmed that the confidence and the commitment are characteristic that are more strengthened recognized in the case of the Coop Italy, since they are more related with the time and with the good performance got, characteristics that that perhaps are already meet in the CCFACC, but not confirmed due to the lack of antiquity. It must be considered that it is a process still in his initial phases and it is difficult to give confidence and commitment to a project not still very known and with small experience.

On the other hand, it is checked that none of the two cases have homogeneity of size; they are cooperatives of different sizes and geographically dispersion. Both have in common that their experiences operate with satisfaction and benefiting all the parts.

Although it has not been verified the homogeneity in physical terms, it has been checked the homogeneity regarding the principles and cooperative values shared, having always like central axioma the human being. In both cases the fundamental reason of office is the sixth cooperative principle "cooperation between cooperatives", betting by a sustainable economy.

Likewise, it is interesting to stand out that some of the results obtained have not previous findings in the literature. On the one hand, regarding the key factors, the main points detected were the following:

- Competitiveness (assorted and price): for the sector of retail specifically, the assortment and the prices are key for their competitiveness. In the literature reviewed it has not been detected as a key factor, it has been confirmed as a problem in previous experiences.

- Marketing homogeneous: The Coop Italy implements it and the results are successful especially giving an image of integrity between the different cooperative supermarkets. The CCFACC also began to implement it recently.

- Own trademark: It has been checked that the commercialization of the own trademark in a central purchasing office is a fundamental tool as a strategy of differentiation and as of tool of profitability for having higher margins. The Coop Italy and the CCFACC have own trademark and a high percentage of the sales belong to this. 
During the literature review, we have detected key factors studied, that neither had mentioned in the literature. In this sense, the following grouping according to his nature were proposed:

- Factors generators of attitudinal behaviors

- Organizational factors

- Commercial factors

- External factors

This structure was useful for the classification of the factors, what allows a more agile reading of the results. Besides, it helped to relate each one of them with the different phases of the cycle of life of a cooperative central purchasing office.

During the research some limitations arose, which were considered in the moment to conclude the study. Best practices and recommendations for a theoretical model of central purchasing office are set only with analysis and the experience of two cases. Although they are two cases selected from a strategic approach, it is considered that only two is a small number to extrapolate the conclusions; being this situation an own characteristic of the methodology used (study of case).

Other limitation of the present study is the reduce time of operation of the CCFACC, question that affects to his experiences and results, considering that these kinds of projects need time of get the results expected.

KEYWORDS: Consumer cooperative, central purchasing, case study. 


\section{Introducción}

En una realidad globalizada, las cooperativas se encuentran actuando en un contexto altamente competitivo, como lo describen Calderwood y Davies (2013), las empresas se encuentran en un "Gran Medio" (precios medios y no diferenciación) y, para destacarse del resto, existen dos estrategias posibles: bajos precios o diferenciación, la dicotomía revelada inicialmente por Porter (1980). Según estos autores, un recurso atractivo para las cooperativas es la aplicación de valores y principios como punto de diferenciación con el resto de las empresas. Además, insisten en que las cooperativas tampoco pueden sumarse a la guerra de precios, ya que su prioridad es mantenerse en funcionamiento para continuar brindando sus servicios, por lo que sus dirigentes deberán saber gestionar adecuadamente las presiones del mercado en el marco de la sostenibilidad, ya que el fin último es la satisfacción de las necesidades de sus asociados. En este sentido, de acuerdo a Brazda y Schediwy (2003), las cooperativas de consumo son una estrategia para los más desfavorecidos.

En este contexto, la innovación se convierte en una estrategia posible para las cooperativas, pero la pregunta es ¿cómo hace un supermercado cooperativo para mostrar que, verdaderamente, es diferente al resto? Una de las posibles soluciones es la aplicación de los principios cooperativos, realizando acciones que las demás cadenas comerciales (no cooperativas) no llevan a cabo. Por ejemplo, realizar campañas solidarias y promover el trabajo colaborativo atraería a los consumidores.

Sin embargo, en el plan para una década cooperativa, la Alianza Cooperativa Internacional $(\mathrm{ACl}$, 2013) expone que el objetivo es la sólida afirmación que la forma de organización diferente de las cooperativas es mejor que la que rige actualmente (empresas con fines de lucro). Se destaca la identidad cooperativa, así como la sostenibilidad y la participación de los socios.

En este sentido, resulta interesante el trabajo de Menzani y Zamagni (2010), ya que estudian las redes cooperativas en la economía italiana y narran las primeras experiencias de asociación entre empresas que tuvieron lugar alrededor de 1970 siendo Italia uno de los países protagonistas. Justamente, en este período de crisis internacional, numerosas cooperativas estaban en riesgo de desaparecer y fue cuando las organizaciones cooperativas de segundo o tercer grado ${ }^{1}$ pusieron empeño en fortalecer el movimiento a través de reformas legislativas, por ejemplo. Dentro de los tipos de asociaciones, las redes horizontales fueron las pioneras y las más comunes, dada la simplicidad de su estructura, y es aquí donde se encuentra el caso de la Coop Italia, como la organización nacional de cooperativas de consumo.

1.- Cooperativa cuyos socios son otras cooperativas o asociaciones de cooperativas, respectivamente. 
Entre las conclusiones del estudio previamente mencionado, se encuentra que el grado de eficiencia de cada tipo de empresa depende del contexto histórico y, dentro de un mundo con diferentes tipos de empresas, hay un lugar para las cooperativas, las cuales "pueden ser perfectamente eficientes en determinadas circunstancias y de ninguna forma deben ser prejuzgadas como inferiores" (Menzani y Zamagni, 2010: 121).

Adicionalmente, Tencati y Zsolnai (2009) desarrollan el interrogante sobre si una competitividad responsable es suficiente y exponen a la Coop Italia como un ejemplo a favor de la hipótesis. La posición distintiva que destacó a la organización fue el verdadero y genuino compromiso de ser sostenible, el cual logró llevar a cabo mediante la aplicación de los principios y valores cooperativos.

Por su parte, Gaeda (2011) presenta una de las mejores adaptaciones del modelo cooperativo al nuevo mercado como es el caso del Grupo Eroski, el cual se basa en dos premisas fundamentales: la rentabilidad entendida en términos de vender muchos productos con márgenes más reducidos $\mathrm{y}$, por otro lado, resaltar una organización en donde tanto trabajadores como consumidores son la esencia del modelo.

Desde la perspectiva argentina, el cooperativismo de consumo remite sus orígenes a fines del siglo XIX gracias a la acción de inmigrantes europeos que desarrollaron sus actividades en forma asociativa y se estima que para el año 1900 el país ya contaba con 56 cooperativas (Montes y Ressel, 2003). Actualmente, el sector presenta una cuota de mercado cercana al 5\%, cuando en 1990 ésta rondaba el 20\% (Masón y Zoppi, 2013). Esta realidad de desigualdad de tasas, junto con el Plan para una Década Cooperativa ( $\mathrm{ACl}, 2013)$, es lo que motiva la implantación de la central de compras virtual, impulsando la cooperación e integración que permitan el crecimiento y desarrollo, en el marco del sexto principio cooperativo.

Cabe destacar que una diferencia crítica entre las empresas privadas y las cooperativas es que, mientras las empresas con fines de lucro persiguen maximizar la ganancia de la firma, las cooperativas buscan maximizar el beneficio tanto de la organización como de cada asociado, entendiendo este último punto como la satisfacción de sus necesidades (Davies y Burt, 2007).

El objetivo de esta investigación es, en primer lugar, detectar los factores clave para el buen funcionamiento de una central de compras cooperativa y, seguidamente, analizar cómo gestiona cada uno de ellos las variables de análisis seleccionadas. El análisis comparativo resulta interesante debido a que se trata de dos casos extremos: por un lado, la central de compras de la Federación Argentina de Cooperativas de Consumo (en adelante, CCFACC), con apenas un año de funcionamiento, y, por el otro, la Coop Italia, un modelo con amplia trayectoria en el mercado, con resultados comprobados y con proyecciones de mayor crecimiento. Un aspecto enriquecedor para el análisis es que cada uno de los casos se ubica en contextos y continentes diferentes. 
El presente trabajo se compone de cinco secciones. En primer lugar, el marco teórico, en donde se realiza una revisión bibliográfica de los factores clave de una central de compras. En segundo lugar, se menciona la metodología utilizada, especificando las unidades de análisis, fuentes de información y análisis de datos. Seguidamente, se exponen los resultados del trabajo, el análisis comparativo sobre cómo afectan los factores clave en cada una de las centrales de compras seleccionadas, y, por último, las conclusiones.

\section{Marco teórico}

El sexto principio cooperativo incita a la cooperación entre cooperativas y la integración es una herramienta clave en este proceso. Según Charterina (2012), este principio es instrumental y de suma importancia para que todas las cooperativas se encuentren en condiciones de cumplir sus objetivos. Además, cobra relevancia en tiempos de crisis.

De acuerdo con lo expuesto por este principio, el movimiento cooperativo trabaja integradamente con estructuras locales, regionales, nacionales e internacionales. Tradicionalmente, las cooperativas conforman agrupaciones de segundo grado (federaciones) y, asimismo, la agrupación de estas últimas da origen a las cooperativas de tercer grado (confederaciones). Estas configuraciones también tienen como objetivo crear transparencia y proveer mecanismos para que las cooperativas, individualmente, puedan adaptarse a un entorno competitivo (Acosta y Verbeke, 2009).

Sobre la integración de compras, tanto Bacic et al. (2008), Acosta y Verbeke (2009) y Regalado et al. (2017) asienten que la cooperación entre empresas es un recurso estratégico y necesario en el mundo empresarial para responder eficazmente a un entorno competitivo. Asimismo, Vuotto (2013) sostiene que uno de los desafíos de la investigación cooperativa es "la integración y la intercooperación para conferir fortaleza en su posicionamiento".

En este sentido, resulta interesante el trabajo de Sanchis Palacio et al. (2015) en donde se describen los factores clave para el desarrollo de cooperativas. En el estudio los autores clasifican los factores entre los conocimientos y habilidades propias de los empresarios y aquellos factores internos de la propia empresa. Como resultado detectan que, dado el reducido tamaño que caracteriza a la mayoría de estas organizaciones, resulta necesario establecer alianzas estratégicas para alcanzar algunos de ellos, tal es el caso de la profesionalización de los dirigentes.

El concepto de "central de compras" se concibe como un mecanismo que permite realizar compras en común, con el objetivo de conseguir mejorar la posición competitiva de sus asociados. De 
acuerdo a Rubio (2017), "las cooperativas de segundo grado constituyen el mecanismo por excelencia de integración cooperativa" (p. 119).

A su vez, según la definición de "central de compras" de la Asociación de Centrales de Compras y Servicios de España (en adelante, ANCECO), éstas garantizan la diversidad de formatos, ya que permiten que las pequeñas y medianas empresas puedan competir al mismo nivel que las grandes empresas (ANCECO, 2016).

Con el fin de comprender el proyecto de una central de compras como un proceso madurativo, Pousa (2006) establece diferentes fases del ciclo de vida de las centrales de compra; estas etapas se distinguen según quién realiza la gestión, el área geográfica en la cual se desempeña, sus objetivos y alguna característica adicional (en la Tabla 1 se desarrolla cada una de las etapas).

Asimismo, en ANCECO (2004) también se asegura que las centrales de compras pasan por distintas fases del ciclo y que en cada una de ellas tienen distintas opciones estratégicas. Se destaca una primera fase de creación, caracterizada por el entusiasmo y el espíritu creativo, seguida por una etapa de madurez, en donde la central ya alcanzó determinada estabilidad y volumen de operaciones. En una tercera etapa se da la diferenciación de los servicios que ofrece la central y se observa una estructura de miembros cada vez más fuerte y homogénea. Luego se da la fase de integración, tanto entre los miembros como entre los proveedores, seguida de un período de optimización en donde tiene lugar la mejora de los procesos. En caso de ser necesaria, se puede llegar a dar una fase de reestructuración de la organización.

Esta segmentación del ciclo de vida de una central servirá para estudiar, sobre todo, el caso italiano, dado que presenta una larga trayectoria en el mercado y su desarrollo fue evolucionando durante estos años. 


\section{Tabla 1. Ciclo de vida de centrales de compras}

\begin{tabular}{|c|c|c|c|c|}
\hline Etapa & Gestión & Área de actuación & Foco & Principal característica \\
\hline Precursora & $\begin{array}{l}\text { Realizada por los propios } \\
\text { asociados }\end{array}$ & Micro regiones & $\begin{array}{l}\text { - Conseguir descuentos } \\
\text { y plazos de compra }\end{array}$ & $\begin{array}{l}\text { - Intercambio de informa- } \\
\text { ción entre los asociados }\end{array}$ \\
\hline Desarrollo & $\begin{array}{l}\text { Realizada por los propios } \\
\text { asociados, con más tiempo } \\
\text { de dedicación }\end{array}$ & Regional & $\begin{array}{l}\text { - Compra conjunta } \\
\text { - Mayor apoyo y benefi- } \\
\text { cio de proveedores }\end{array}$ & $\begin{array}{l}\text { - Razonable grado de } \\
\text { integración de informa- } \\
\text { ción entre los asociados } \\
\text { - Incorporación de servi- } \\
\text { cios de marketing y fi- } \\
\text { nanzas }\end{array}$ \\
\hline Maduración & $\begin{array}{l}\text { Dedicación exclusiva de } \\
\text { empresario o personal con- } \\
\text { tratado }\end{array}$ & Varias regiones & $\begin{array}{l}\text { - Ampliación de los recur- } \\
\text { sos ofrecidos de mar- } \\
\text { keting, finanzas y recur- } \\
\text { sos humanos }\end{array}$ & $\begin{array}{l}\text { - Formalización de pro- } \\
\text { cesos } \\
\text { - Mejora de la logística, } \\
\text { posible abastecimiento } \\
\text { centralizado }\end{array}$ \\
\hline Madurez plena & $\begin{array}{l}\text { Gestión altamente profesio- } \\
\text { nal, con sólidos conocimien- } \\
\text { tos en planeamiento estra- } \\
\text { tégico }\end{array}$ & $\begin{array}{l}\text { Nacional o varias } \\
\text { regiones }\end{array}$ & $\begin{array}{l}\text { - Integración tecnológica } \\
\text { con proveedores } \\
\text { - Sistemas de informa- } \\
\text { ción } \\
\text { - Logística integrada } \\
\text { - Pagos centralizados }\end{array}$ & $\begin{array}{l}\text { - Se puede llegar a la } \\
\text { creación de empresas, } \\
\text { en donde los socios tie- } \\
\text { nen participación accio- } \\
\text { naria }\end{array}$ \\
\hline
\end{tabular}

FUENTE: Pousa (2006) en base a Souza (2004).

De acuerdo con el objetivo de llevar a cabo un relevamiento de los factores clave que contribuyen al desarrollo de las centrales de compras, se analizaron tres estudios que, precisamente, investigan los factores de mayor incidencia en el exitoso funcionamiento. Es posible que en muchos casos los factores clave tengan relación con los problemas detectados, hecho que ratifica la importancia de la buena práctica.

En primer lugar, Hoffmann y Schlosser (2001) realizan un estudio sobre los factores de éxito en las alianzas estratégicas entre pequeñas y medianas empresas, específicamente en 164 empresas austríacas, y determinan que un factor clave es aquel que define el éxito o fracaso de una alianza. En este caso, los ocho factores críticos que encontraron fueron:

- Definición precisa de derechos y obligaciones

- Contribución con determinadas fortalezas que puedan aportar sinergia

- Conciencia de requerimiento de tiempo

- Contribuciones equitativas para todos los participantes 
- Puesta a disposición de la red de los servicios y/o recursos requeridos

- Derivación de los objetivos de la red de la estrategia comercial

- Construcción de la confianza a través de la unificación para evitar el oportunismo

- Implementación ágil y rápidos resultados

Los autores concluyen en que tanto las variables referidas al contenido como a los procesos son importantes para el desempeño de una alianza. A su vez, afirman que las buenas relaciones interpersonales y la confianza entre los participantes son prerrequisitos para la cooperación.

En el segundo caso, Torres Ruiz et al. (2006) detallan los factores decisivos para el éxito de centrales de compras en el cooperativismo agrario español, con el objeto de mejorar las condiciones en la obtención de inputs y desarrollar determinados servicios compartiendo costos o alcanzando una escala suficiente para un mejor resultado. Las principales recomendaciones que establecen dichos autores son:

- Independencia y justificación de la central de compras desde el punto de vista económico

- Establecimiento de un modelo secuencial de crecimiento

- Equilibrio entre la flexibilidad y compromiso

- Gestión profesional que genere confianza y comunique

- Búsqueda continua de mayor dimensión

- Búsqueda de la fidelización del socio mediante la diversificación, el desarrollo de programas l+D y la especialización en servicios

- Gestión participada para conseguir mayor implicación y confianza de los socios

Por su parte, Lagemann (2004) también se propone buscar los factores que influencian la performance de las redes de compras de pequeñas y medianas empresas a través de un trabajo de campo con 20 redes brasileñas de Río Grande do Sul. Tras una extensa revisión bibliográfica, concluye que los factores clave son:

- Confianza

- Compromiso

- Aprendizaje (intercambio de información)

- Experiencia

- Compatibilidad (sinergia y resolución de conflictos)

- Equilibrio de derechos y deberes

- Capacitación de recursos humanos

- Procesos de formación y estructura

- Coordinación y mecanismos de control

- Ambiente externo 
Tras el análisis y síntesis de la información, es posible listar las variables estudiadas:

\section{Confianza}

Las tres investigaciones coinciden en que es fundamental la confianza, tanto en el sentido de confianza entre los participantes para evitar hechos de oportunismo, como la necesidad de construir confiabilidad en el modelo. En este sentido, también es sumamente importante que los asociados tengan conciencia del valor de la cooperación, o sea, confíen en el modelo para poder lograr el objetivo conjunto.

Adicionalmente, Torres Ruiz et al. (2006) justifica que una gestión profesional de la central es necesaria para generar confianza y comunicar, así como para conseguir resultados visibles y convencer a la masa de socios.

\section{Compromiso}

Según Lagemann (2004), el compromiso se relaciona directamente con la lealtad, por lo que se transforma en un factor determinante de un proyecto. Es posible identificar dos tipos de compromisos; uno referido a lo actitudinal, la voluntad y el esfuerzo que conlleva una participación activa, y; por otro lado, un compromiso que apoye los beneficios esperados por la central.

Por otro lado, Hoffmann y Schlosser (2001) también mencionan la necesidad de conciencia por parte de los socios de que la participación en este tipo de proyectos les va a demandar tiempo y es necesaria su predisposición, así como la disponibilidad de sus recursos para otros participantes y la central misma.

\section{Gestión participada}

Es necesario que una representación del colectivo participe en la toma de decisiones de la central y que sus opiniones sean tenidas en cuenta. De esta forma, se logrará mayor involucración y sentimiento de identidad con el proyecto (Torres Ruiz et al., 2006).

\section{Compatibilidad y complementariedad}

Considerando que es imposible que las empresas sean homogéneas y las sinergias sean perfectas, se busca el mayor grado de compatibilidad posible, para lo cual es necesario que cada una de las organizaciones posea habilidades para trabajar en conjunto. A su vez, Hoffmann y Schlosser 
(2001) afirman que es necesario que cada socio presente alguna fortaleza que pueda contribuir de manera positiva a la sinergia del grupo y, por otra parte, que los objetivos del proyecto y los individuales de cada organización estén alineados.

De todas maneras, las diferencias entre los participantes no son deseables en este tipo de proyectos, en donde debe regir la equidad y la transparencia. Hoffmann y Schlosser (2001) agregan que la reciprocidad entre los socios es fundamental para mejorar la performance de la central y que una dependencia estratégica de la central determina el poder de la estructura.

\section{Modelo secuencial de crecimiento}

Más allá de si los resultados son en el corto 0 en el largo plazo, es necesario contar con un modelo secuencial de crecimiento, tal como explica Torres Ruiz et al. (2006), para que todos se encuentren alineados hacia la búsqueda de resultados. El modelo secuencial de crecimiento será de utilidad para el marketing interno de la organización.

La implementación ágil y los resultados rápidos que mencionan Hoffmann y Schlosser (2001) terminan siendo consecuencia de un modelo diseñado correctamente para cada caso posible de ser implementado sin dificultades.

\section{Búsqueda continua de mayor dimensión}

Según Torres Ruiz et al. (2006), resulta necesario el crecimiento continuo del volumen de compra para conseguir mejores condiciones de negociación y precios más competitivos para los socios.

\section{Independencia económica de la central}

Torres Ruiz et al. (2006) sostienen que el sector debe percibir la necesidad y la conveniencia del proyecto, a la vez que el sector público puede y debe apoyarlo, pero no implicarse en exceso. En este sentido, Adobor y McMullen (2010) afirman que la central debe cumplir el rol de puente altruista, a la vez de ser un actor activo, imparcial y honesto.

Es posible relacionar este concepto con el cuarto principio cooperativo "Autonomía e Independencia" haciendo alusión, principalmente, a la independencia a la hora de la toma de decisiones. A su vez, se puede decir que este principio se encuentra inspirado en las primeras reglas de Rochdale, dado que sólo estaba permitido vender al contado porque veían al crédito como un mal social. 


\section{Capacitación y aprendizaje}

La capacitación se vuelve fundamental para sostener las alianzas estratégicas, por eso es necesario contratar profesionales especializados para contar con el conocimiento adecuado del área.

Además, afirma Lagemann (2004), es necesario que la red cuente con personal dedicado exclusivamente a la gestión de la central y que se capaciten constantemente. Hoffmann y Schlosser (2001) agregan que cuanto mayor sea la capacidad de aprender y la transparencia del conocimiento complementario de los asociados, mayor será el éxito de la capacitación.

Al concepto de capacitación también se le puede asociar el de "procesos" que, según Lagemann (2004), refiere a la capacidad de la central de brindar herramientas de apoyo para asegurar la comunicación y la buena gestión de cada una de las organizaciones. En tal sentido, la contribución de internet es fundamental, ya que se pueden diseñar sistemas para ayudar en los procesos a los que tengan acceso todos los participantes.

Por otro lado, un aspecto no menor es la formación cooperativa. Barberini (2009) hace hincapié en promover las ideas del movimiento a los jóvenes; teniendo en cuenta que los cambios generacionales son inminentes, los dirigentes con experiencia deben contribuir a prevenir errores.

\section{Comunicación}

La comunicación es primordial en este tipo de proyectos: el intercambio de información debe ser fluido y recíproco. La central tiene que proporcionar todo tipo de información a los asociados para asegurar su transparencia en un 100\% (Adobor y McMullen, 2010), a la vez que los asociados deben aportar información a la central y entre ellos también, para aprender de las experiencias pasadas y mejorar el desempeño de cada uno (Lagemann, 2004).

\section{Experiencia}

Es de utilidad para el proyecto que tanto el personal que gestiona la central como los asociados tengan experiencia en redes colaborativas y puedan realizar aportes para lograr mejoras.

\section{Claridad de derechos y obligaciones}

Lagemann (2004) y Hoffmann y Schlosser (2001) coinciden en que es necesaria una clara definición de derechos y obligaciones de cada parte para no tener conflictos, 0 sea, reglas claras desde 
el principio. El equilibrio entre los asociados es la prioridad, y todos deben tener derecho a voto, igualdad de voto y posibilidad de ser elegidos.

\section{Coordinación y mecanismos de control}

Resulta necesario que la red fiscalice las acciones de los asociados para asegurarse el cumplimiento de los objetivos comunes. Como resultado de la revisión bibliográfica, Lagemann (2004) encuentra que algunas alternativas serían rutinas interorganizacionales, prácticas comunes de sector, contratos 0 acuerdos escritos.

\section{Ambiente externo}

De acuerdo con Lagemann (2004), existen factores externos que afectan directamente a la performance de un proyecto, como las políticas públicas y la aceptación de los distintos actores del proceso (proveedores, competidores, clientes); que también definen el desempeño del modelo.

En este sentido, Bastidas-Delgado (2015) expone diferentes experiencias a nivel mundial sobre cómo ha influido el contexto, tanto positiva como negativamente, en el desarrollo del cooperativismo. Por ejemplo, Rusia, China o Cuba se han nutrido del modelo cooperativo para llevar adelante su proyecto de gobierno, siendo Venezuela el caso más actual. Por otro lado, se reconoce la relevancia de la relación con los sindicatos, teniendo en cuenta que una propuesta interesante impulsada por la Organización Internacional del Trabajo (OIT), la ACl y la Confederación de Sindicatos Libres pretende profundizar las alianzas entre sindicatos y cooperativas, con el objetivo de organizar a los trabajadores de la economía informal a través de la solidaridad y de la asistencia mutua.

\section{Metodología}

La metodología utilizada en la investigación fue el estudio de casos múltiple, de casos cruzados o entrelazados, debido a que, desde el principio del estudio, los investigadores pretendieron revisar comparativamente las unidades de análisis entre sí buscando similitudes y diferencias (HernandezSampieri, et al. 2010)

Se seleccionaron los casos, como estudios extremos; una central de compras cooperativa con 50 años de funcionamiento y una creada hace dos años. Sin embargo, ambas centrales son entidades 
de grado superior que nuclean a las cooperativas de primer grado y se rigen por los valores y principios cooperativos.

Yin (2001) argumenta que las unidades de análisis, las fuentes de información y el análisis de datos son componentes fundamentales del estudio de caso, los cuales se desarrollarán a continuación.

\subsection{Unidades de análisis}

Casal y Mateu (2003) aseguran que el muestreo se determina por conveniencia, dado que la selección de las unidades de análisis no es aleatoria, se seleccionan por ser casos típicos (Suárez et al., 2004).

Por lo tanto, este estudio cuenta con dos unidades de análisis seleccionadas por ser casos reveladores y, a priori, ejemplos extremos que pueden resultar en un enriquecedor análisis comparativo.

Por un lado, la central de compras de la Federación Argentina de Cooperativas de Consumo (CCFACC), la central de compras cooperativa argentina transitando sus inicios de funcionamiento, $y$, por el otro, la Coop Italia, un modelo desarrollado y afianzado en el mercado, con resultados comprobados y con proyecciones de expansión. El análisis comparativo de estas dos centrales resulta interesante por tratarse de dos proyectos en etapas diferentes y en contextos y continentes distintos. En el Tabla 2 se comparan los aspectos más relevantes de las dos centrales.

\subsection{Fuentes de información}

Distintas fuentes de información fueron utilizadas en el presente trabajo como documentación interna de ambas entidades, boletines, registros e informes de gestión de ventas y asociados, presentaciones y dossiers, entre otros.

Asimismo, en septiembre de 2016 se llevó a cabo en Bahía Blanca (Argentina) el Encuentro Intercooperativo organizado por la propia FACC, donde tanto las cooperativas de consumo, como de producción y trabajo, tuvieron lugar para comentar sus experiencias y sugerir mejoras para el funcionamiento de la central de compras.

Por otra parte, también se han realizado entrevistas a representantes de la CCFACC (Presidente, Secretario y Consejero) y de la Coop Italia (Vice Presidente Vicario ANCC-COOP), cuando visitaron personalmente las distintas entidades. A su vez, se tomó como fuente de información secundaria la entrevista a Barberini (2009), cooperativista italiano de referencia a nivel mundial, ex presidente de la Coop Italia y de la $\mathrm{ACl}$. 


\section{Tabla 2. Características relevantes de la CCFACC y Coop Italia}

\begin{tabular}{|c|c|c|}
\hline Característica & CCFACC & Coop Italia \\
\hline Fundación & 2016 & 1968 \\
\hline Socios & $\begin{array}{l}24 \text { cooperativas de consumo o con sección con- } \\
\text { sumo - } 1 \text { cooperativa grande, } 1 \text { mediana y las } \\
\text { demás pequeñas (por lo menos la sección con- } \\
\text { sumo) }\end{array}$ & $\begin{array}{l}91 \text { cooperativas de consumo }-7 \text { grandes coo- } \\
\text { perativas, } 8 \text { medianas y } 76 \text { pequeñas }\end{array}$ \\
\hline Dispersión geográfica & Territorio argentino (2,78 millones de km2) & Territorio italiano (300.000 km2) \\
\hline Puntos de venta & 198 locales & 1.165 locales \\
\hline $\begin{array}{l}\text { Participación de mer- } \\
\text { cado }\end{array}$ & $5 \%$ (estimado) & $14,5 \%$ del total de ventas de retail \\
\hline Organización & Administración central a cargo de la FACC. & $\begin{array}{l}\text { Administración central y consorcios regionales } \\
\text { (agrupaciones de cooperativas) }\end{array}$ \\
\hline $\begin{array}{l}\text { Integración internacio- } \\
\text { nal }\end{array}$ & $\mathrm{N} / \mathrm{A}$ & $\begin{array}{l}\text { Alianza con Coopernic (4 redes cooperativas } \\
\text { europeas) }\end{array}$ \\
\hline Gestión de pedidos & Portal virtual. Total de pedidos consolidados. & Portal virtual. \\
\hline Gestión de compras & $\begin{array}{l}\text { La negociación con los proveedores está a cargo } \\
\text { de la cooperativa más importante. }\end{array}$ & $\begin{array}{l}\text { La Coop negocia con los proveedores más impor- } \\
\text { tantes y los consorcios lo hacen directamente con } \\
\text { los proveedores regionales o más pequeños. }\end{array}$ \\
\hline Surtido & Almacén, perecederos, perfumería, bazar y hogar & $\begin{array}{l}\text { Almacén, perecederos, perfumería, bazar, tienda } \\
\text { y hogar }\end{array}$ \\
\hline Marca propia & $\begin{array}{l}120 \text { referencias - Cooperativa Obrera es el } \\
\text { segundo proveedor más importante de la central. }\end{array}$ & $\begin{array}{l}7 \text { líneas de productos - Más de } 4.000 \text { referencias } \\
-26 \% \text { de incidencia. }\end{array}$ \\
\hline Marketing & $\begin{array}{l}\text { Acciones centralizadas (especial productos coo- } \\
\text { perativos), las demás las maneja cada coopera- } \\
\text { tiva. }\end{array}$ & $\begin{array}{l}\text { Acciones calendario centralizadas, las demás las } \\
\text { maneja cada consorcio/cooperativa. }\end{array}$ \\
\hline Logística & $\begin{array}{l}\text { Sin depósitos ni logística propia. La logística } \\
\text { depende de cada cooperativa asociada. }\end{array}$ & $\begin{array}{l}\text { Cada consorcio tiene depósitos propios. } \\
\text { Tercerizan la logística a través de una coopera- } \\
\text { tiva de transporte, aunque la dirección está a } \\
\text { cargo de la Coop. }\end{array}$ \\
\hline $\begin{array}{l}\text { Otros servicios ofreci- } \\
\text { dos }\end{array}$ & $\begin{array}{l}\text { Marketing, gestión de supermercados, diseño de } \\
\text { layouts. }\end{array}$ & $\begin{array}{l}\text { Marketing, diseño de layouts, preparación de } \\
\text { contratos con proveedores. }\end{array}$ \\
\hline
\end{tabular}

FUENTE: Elaboración propia. 
Complementariamente, en las visitas a las entidades, también se efectuó la observación directa.

Por último, como es posible observar, se utilizaron diversas fuentes información para garantizar la triangulación de las fuentes de datos (Villareal y Landeta, 2010).

\subsection{Análisis de datos}

Con el objetivo de analizar cómo interviene cada factor clave determinado en cada una de las unidades de análisis seleccionadas, se ha trabajado con el material recolectado durante los foros, con la información interna y la obtenida en las entrevistas a representantes de las centrales, realizando un análisis cualitativo y tratando de sintetizar la misma de manera objetiva.

En relación al análisis cualitativo de los datos, el mismo se ha realizado siguiendo los pasos detallados por Ryan y Bernard (2003):

- Obtención de la información: registro sistemático de notas de campo, entrevistas y documentación interna.

- Captura, transcripción y ordenamiento: en el caso de la documentación interna, la recolección del material original, $y$, en el caso de las entrevistas y foros, captura a través de grabaciones y posterior transcripción.

- Codificación: proceso en el cual se agrupa la información por categorías que concentran temas similares. Dichos códigos se utilizan para ordenar de forma más útil la información.

- Integración de la información: agrupación de la información por categorías entre sí y con los fundamentos teóricos hallados previamente.

\subsection{Resumen de la metodología implementada}

La Tabla 3 presenta las decisiones tomadas relativas a la metodología escogida para el presente estudio. 


\section{Tabla 3. Resumen de la metodología implementada}

\begin{tabular}{|l|l|l|}
\hline Ámbitos de decisión & Decisión adoptada & Descripción \\
\hline $\begin{array}{l}\text { Estrategia de la inves- } \\
\text { tigación }\end{array}$ & Estudio de caso múltiple cruzado. & $\begin{array}{l}\text { El propósito del estudio es conocer los factores clave para } \\
\text { el buen funcionamiento de una central de compras coo- } \\
\text { perativa. Desde el principio se han comparado los casos } \\
\text { buscando las similitudes y diferencias. }\end{array}$ \\
\hline $\begin{array}{l}\text { Selección de los } \\
\text { casos }\end{array}$ & $\begin{array}{l}\text { Selección de casos extremos basado en } \\
\text { la oportunidad de la obtención de la infor- } \\
\text { mación }\end{array}$ & $\begin{array}{l}\text { Se escogió la COOP Italia y la CCFACC. La primera cen- } \\
\text { tral con más de 50 años de trayectoria y la segunda una } \\
\text { experiencia nueva en pleno proceso de consolidación. }\end{array}$ \\
\hline Recolección de datos & $\begin{array}{l}\text { Técnicas e instrumentos cualitativos de } \\
\text { recolección de datos }\end{array}$ & $\begin{array}{l}\text { Observación directa, entrevistas semi estructuradas e } \\
\text { información secundaria (documentación interna de ambas } \\
\text { entidades, boletines, registros e informes de gestión de } \\
\text { ventas y asociados, presentaciones y dossiers). }\end{array}$ \\
\hline Análisis de datos & $\begin{array}{l}\text { Aplicar técnicas de análisis de datos pro- } \\
\text { puestas por Ryan y Bernard (2003) }\end{array}$ & $\begin{array}{l}\text { Etapas de análisis: obtención de la información; captura, } \\
\text { transcripción y ordenamiento; codificación e integración } \\
\text { de la información. } \\
\text { Se utilizó el software NVIVO11 para realizar el análisis de } \\
\text { la información. }\end{array}$ \\
\hline
\end{tabular}

FUENTE: Elaboración propia.

\section{Resultados}

Con el objetivo de analizar los factores clave hallados en la literatura, siguiendo la dinámica del trabajo de Sanchis Palacio et al. (2015), se propone clasificarlos para una organización más adecuada de la información y una mejor comprensión. Seguidamente, se estudian los mismos agrupándolos en factores generadores de conductas actitudinales, organizacionales, comerciales y externos. En la Tabla 4 es posible apreciar dicha clasificación: 


\section{Tabla 4. Clasificación de factores clave de éxito para central de compras cooperativa}

\begin{tabular}{|l|l|l|}
\hline Clasificación & Descripción & Factor clave \\
\hline $\begin{array}{l}\text { Generadores de con- } \\
\text { ductas actitudinales }\end{array}$ & $\begin{array}{l}\text { Factores que determinan la posición de las cooperativas } \\
\text { asociadas ante el proyecto. }\end{array}$ & $\begin{array}{l}\bullet \text { Confianza } \\
\bullet \text { Compromiso } \\
\bullet \text { Gestión participada }\end{array}$ \\
\hline Organizacionales & $\begin{array}{l}\text { Factores propios de gestión, que pueden ir variando } \\
\text { dependiendo del progreso del proyecto. }\end{array}$ & $\begin{array}{l}\bullet \text { Compatibilidad y complementariedad } \\
\bullet \text { Capacitación y aprendizaje } \\
\bullet \text { Comunicación } \\
\bullet \text { Experiencia } \\
\text {-Claridad de derechos y obligaciones } \\
\bullet \text { Coordinación y mecanismos de control }\end{array}$ \\
\hline Comerciales & $\begin{array}{l}\text { Factores relacionados a la actividad comercial tendientes } \\
\text { a lograr la eficiencia. }\end{array}$ & $\begin{array}{l}\bullet \text { Modelo secuencial de crecimiento } \\
\text { Búsqueda de mayor dimensión } \\
\bullet \text { Independencia económica de la central }\end{array}$ \\
\hline Externos & $\begin{array}{l}\text { Variables exógenas relacionadas con el contexto que } \\
\text { influyen en el proyecto. }\end{array}$ & $\bullet$ Ambiente externo \\
\hline
\end{tabular}

FUENTE: Elaboración propia.

En segundo lugar, la Tabla 5 (página siguiente) expone los resultados por factor clave en cada una de las dos centrales.

Primeramente, se destaca como factor crítico la confianza, la que se va ganando endógenamente y que sólo la maduración del proyecto puede brindar. Por lo tanto, en el caso de la CCFACC, es un aspecto que aún está en proceso, debido a que están en la fase inicial del ciclo, en donde la incertidumbre tiene lugar. Por ejemplo, un importante número de cooperativas esperó a que se pusiera en marcha la central y luego se asociaron, o participan, pero no realizan todas las compras posibles a través de la central. Según el secretario de la CCFACC, "para nosotros la confianza es un obstáculo a vencer y va a requerir la capacidad de convicción de nuestra gente, la capacidad de convencer a los cooperativistas que esto es bueno para todos".

Distinta es la realidad de la Coop Italia, la cual, gracias a los años que hace que está en funcionamiento, se ha ganado la confianza de sus socios y se posiciona en un lugar privilegiado dentro del mercado italiano. Un indicador que demuestra la confianza de los asociados hacia el modelo es el porcentaje de compras que se realizan a través de la central, el cual es del $98 \%$ aproximadamente, por lo cual casi la totalidad de las compras de los socios son mediante la Coop. 


\section{Tabla 5. Incidencia de factor clave en CCFACC y Coop Italia}

\begin{tabular}{|c|c|c|}
\hline Factor clave & CCFACC & Coop Italia \\
\hline \multicolumn{3}{|c|}{ Factores generadores de conductas actitudinales } \\
\hline Confianza & En desarrollo. & El $98 \%$ de las compras pasan por la central. \\
\hline Compromiso & $\begin{array}{l}\text { En desarrollo. Existencia de cooperativas con } \\
\text { sección consumo (el consumo no es su mayor } \\
\text { preocupación). }\end{array}$ & Cooperativas de consumo más comprometidas. \\
\hline Gestión participada & Encuentros periódicos. & Participación de las cooperativas en consorcios. \\
\hline \multicolumn{3}{|c|}{ Factores organizacionales } \\
\hline $\begin{array}{l}\text { Compatibilidad y com- } \\
\text { plementariedad }\end{array}$ & Dispersión geográfica y diferencia de tamaños. & Dispersión geográfica y diferencia de tamaños. \\
\hline $\begin{array}{l}\text { Capacitación y apren- } \\
\text { dizaje }\end{array}$ & Capacitaciones periódicas. & Scuola Coop. \\
\hline Comunicación & $\begin{array}{l}\text { Comunicación en portal web. Documentación } \\
\text { interna/pública. }\end{array}$ & $\begin{array}{l}\text { Comunicación en portal web. Documentación } \\
\text { interna/pública. }\end{array}$ \\
\hline Experiencia & $\begin{array}{l}\text { Experiencia anterior en Primer Precio. Folders } \\
\text { "Especial Cooperativas". }\end{array}$ & $\begin{array}{l}\text { La experiencia nacional le sirve para una inte- } \\
\text { gración internacional. }\end{array}$ \\
\hline $\begin{array}{l}\text { Claridad de derechos } \\
\text { y obligaciones }\end{array}$ & Contratos escritos. & Contratos escritos. \\
\hline $\begin{array}{l}\text { Coordinación y meca- } \\
\text { nismos de control }\end{array}$ & Contratos escritos. & Contratos escritos. \\
\hline \multicolumn{3}{|c|}{ Factores comerciales } \\
\hline $\begin{array}{l}\text { Modelo secuencial de } \\
\text { crecimiento }\end{array}$ & Estrategia de crecimiento secuencial. & $\begin{array}{l}\text { Ha transcurrido por diferentes etapas del ciclo de } \\
\text { vida de una central de compras; ya se encuentra } \\
\text { en una fase de maduración. }\end{array}$ \\
\hline $\begin{array}{l}\text { Búsqueda de mayor } \\
\text { dimensión }\end{array}$ & Crecimiento proyectado. & $\begin{array}{l}\text { Comercio exterior. Alianza con Coopernic. } \\
\text { Alianza 3.0. }\end{array}$ \\
\hline $\begin{array}{l}\text { Independencia econó- } \\
\text { mica de la central }\end{array}$ & $\begin{array}{l}\text { Tasa de sostenimiento. El resultado de la central } \\
\text { debe ser cero. }\end{array}$ & $\begin{array}{l}\text { Tasa de sostenimiento. El resultado de la central } \\
\text { debe ser cero. }\end{array}$ \\
\hline \multicolumn{3}{|c|}{ Factores externos } \\
\hline Ambiente externo & $\begin{array}{l}\text { Políticas públicas, otras centrales de compras y } \\
\text { red de distribución logística preestablecida }\end{array}$ & Políticas públicas \\
\hline
\end{tabular}


En este sentido, es posible relacionar la confianza en el modelo con el compromiso hacia el mismo. Cuanto mayor confianza se tenga en que el participar del proyecto es beneficioso, mayor será el compromiso de cada uno, así también como el esfuerzo destinado para el buen funcionamiento. Un caso particular que se da en la central argentina es que en la mayoría de las cooperativas participantes el consumo no es su sector principal, son cooperativas de otra naturaleza (agrícolas, ganaderas, de servicios, entre otras) con sección consumo, la cual, muchas veces, está descuidada y su manejo no está totalmente profesionalizado, por lo que el compromiso y la importancia que se le da a este tipo de proyectos no es la adecuada. De acuerdo a lo relatado por el consejero de la central, en algunos casos, parece importar más la cuestión comercial que la integración cooperativa: "Esto es una conjunción de principios y valores, y uno de los principios es la integración, pero en algunos casos prima el pragmatismo".

En cambio, en el caso de la Coop Italia, las cooperativas son de consumo y están sumamente interesadas en participar y en colaborar con el proyecto, por lo que mantienen una participación más activa, sobre todo, dentro de los consorcios.

En relación a la gestión participada, el vicepresidente vicario de la Coop sostiene: "hay cooperativas que forman parte del consorcio, que participan y tienen representación en el consorcio, y después están las cooperativas adheridas, que no llegan a formar parte del consorcio, pero sí tienen todo el know how y toda la estructura del local en base a la imagen marca de los productos que abastece la central, es otra modalidad de negociación".

En la CCFACC, actualmente la participación se da en los encuentros intercooperativos, en donde se llevan a cabo tanto conferencias como foros en donde los participantes pueden plantear sus consultas y sugerencias. La administración de la central tiene en cuenta los temas tratados en estos encuentros y después actúa en búsqueda soluciones y mejoras. Por ejemplo, en un encuentro realizado durante el 2016 surgió la necesidad de contar con surtido de perecederos, que no había en aquel momento, y recientemente se acaba de incorporar una línea de productos perecederos como un primer paso para solucionar la demanda de los socios.

Dos características en común que presentan ambas centrales es la dispersión geográfica y los diferentes tamaños de las cooperativas asociadas. Asimismo, estos aspectos no han sido impedimento para la compatibilidad entre los participantes. Por ejemplo, en el caso italiano se observa que se han agrupado regionalmente y han establecido centros logísticos propios de cada consorcio, una demostración de la complementariedad entre los diferentes tamaños.

En realidad, uno de los fundamentos del proyecto de una central de compras es la complementariedad, razón por la que se da la consolidación de los pedidos en las dos unidades de análisis seleccionadas. En el caso de la CCFACC, la Cooperativa Obrera (la más grande) aporta a la central su poder de negociación con los proveedores y la gestión de las compras en sí, cuestión que sería más complicada para las cooperativas pequeñas sin este apoyo. 
De acuerdo a un modelo secuencial de crecimiento, la CCFACC cuenta con una estrategia de crecimiento y tiene objetivos tanto a corto como a largo plazo. Según el presidente de la FACC, debe haber un desarrollo armónico y gradual, pues si hay comprensión de la importancia que tiene el proyecto, este desarrollo se produciría más rápido. Es posible afirmar que hay una estrategia implícita de crecimiento, dado que se identifica que un objetivo a corto plazo es reunir el $10 \%$ del total de ventas de las cooperativas asociadas.

Por otra parte, es posible detectar que la central italiana ha tenido distintas etapas a lo largo de su ciclo de vida: "La primera etapa consistió en la consolidaron para comprar, ahí se hizo hincapié en marketing, imagen marca y desarrollo de marca propia, por ejemplo. Después se pasó al estadío de consorcios regionales, etapa de optimización económica para que las cooperativas deleguen esas actividades. A su vez, ahora está pasando que muchas de las cooperativas, por el bajo nivel que tienen, solas no pueden subsistir, entonces están en la etapa de consolidación de cooperativas y, por otra parte, se tiene el comercio internacional como el principal foco de crecimiento para el futuro próximo", según aclara el vicepresidente vicario de la Coop.

Al mismo tiempo, la realidad del modelo secuencial de crecimiento en los dos casos se puede relacionar con la búsqueda continua de mayor dimensión, la CCFACC busca aumentar cada vez más su surtido, el número de cooperativas asociadas y el porcentaje de cobertura de las compras de sus asociadas, mientras que el objetivo de la Coop Italia está puesto en el comercio internacional, tanto en las alianzas con otras redes de otras nacionalidades como en la comercialización a nivel de la marca propia en otros países.

El surtido se expuso como una expectativa tanto para las cooperativas como para la CCFACC. A corto plazo se espera llegar al $40 / 50 \%$ de cobertura en los primeros meses de la central, mientras que el objetivo a largo plazo es cubrir las familias de producto que conforman el $80 \%$ de la venta de un supermercado. En principio, se pretendió cubrir todo el surtido con productos de origen cooperativo, pero ello no es posible actualmente debido a que no existen cooperativas de producción que comercialicen todo el surtido. Por lo tanto, la central se ha visto obligada a incorporar productos de otras empresas para facilitar un mejor servicio a sus cooperativas adheridas.

Transcurrido un año de funcionamiento, las cooperativas toman al surtido como un beneficio derivado de su participación en la central. El hecho de poder comercializar los productos de la central permitió poder llegar a los asociados con un mayor surtido y a precios más competitivos. En lo que respecta a la oferta de artículos del hogar, se valora la posibilidad de contar con artículos marca propia que, más allá de presentar un precio competitivo, también contribuye a la fidelización de los asociados. Por otro lado, cooperativas que antes no contaban con el sector de hogar, actualmente ampliaron su surtido y están ofreciendo productos para el hogar marca propia.

En contraposición a la imagen cooperativa convencional por parte de los consumidores, un aspecto a destacar es la calidad de los productos cooperativos, debido a que los productos de origen coope- 
rativo son sinónimo de productos saludables y acordes con una economía sostenible. Por una parte, esta característica de los productos es una expectativa por parte de las cooperativas, para poder ofrecerles a sus asociados productos de buena calidad a un bajo precio; por otra parte, constituye un desafío para la CCFACC. Lograr los estándares de calidad requiere cumplir con ciertas normas y requisitos formales, exigencia que hoy es costosa para algunas de las cooperativas de producción, pero que se puede visualizar como una oportunidad y garantía de aptitud para la venta. En el caso argentino, la posibilidad consiste en modernizarse y actualizarse en cuanto a los requerimientos legales, visualizando la oportunidad de multiplicar sus productos, diversificarlos y mejorar su packaging; en otras palabras, ser más competitivos.

En relación a la homogeneización, también se observa la particularidad de la marca propia en los dos casos, la cual se encuentra en constante crecimiento en ambos casos. En la Coop Italia, ésta ya cuenta con más de 4.000 referencias, abarcando la totalidad de familias de productos, y acumula el $26 \%$ de las ventas (Migliavacca, 2016). Por el lado de la CCFACC, también es importante la marca propia por contar con más de 120 referencias y tener proyecciones de seguir incorporando más productos y llegar a representar el $40 \%$ de las ventas de la Central. Según el consejero de la central: "Un aspecto a mejorar es el surtido y la cantidad de productos marca propia a incorporar, dado que es un valor importante que podemos aportar".

"El resultado de la central debe ser cero", son las palabras del vicepresidente vicario de la Coop, por lo que se entiende que la tasa pagada por los socios es meramente para sostener el funcionamiento de la misma. Igual situación sucede en la CCFACC, en donde se abona un mínimo porcentaje para subvencionar el funcionamiento. En ningún caso se observa que el objetivo sea obtener lucro con las centrales, si el funcionamiento integral es eficiente, todos ganan. El Estado, en ninguno de los dos ejemplos, tiene un papel relevante, coincidiendo con lo expuesto por el cuarto principio cooperativo que hace alusión a la Autonomía e Independencia.

En relación a la capacitación, la estructura de la Coop Italia contempla una organización independiente destinada específicamente a temas de capacitación, la cual se denomina Scuola Coop. Según el vicepresidente vicario de la Coop, existen dos tipos de formación: escuela cooperativa nacional (a mandos medios y altos) y formación para las nuevas incorporaciones con el objetivo de formarlos en cooperativismo y para que conozcan en qué parte del sistema empiezan a trabajar.

Un aspecto importante que también se señala es la formación sobre la gestión del sistema distributivo en relación a los cambios de consumo. El consumo alimentario en los años 70 representaba el $30 \%$ y hoy representa el 12/13\%. Es un consumo más flexible, ya que hay más información. Estos aspectos son los que hacen dinámico el mercado y exigen la necesidad de formación constante.

Por su parte, la CCFACC ofrece formación periódicamente en diferentes puntos geográficos del país, para facilitar el acceso de todos los asociados. La selección de temas se realiza teniendo en 
cuenta las sugerencias de los asociados y se busca especialistas en cada área. A su vez, también se brinda material a demanda vía web.

Por otro lado, referido a los procesos, en ambas centrales se encuentra que se brindan servicios de gestión de supermercados, diseño de layouts y marketing, entre otros. La Coop Italia cuenta con un área específica de apoyo de estos temas a las cooperativas adheridas.

Siguiendo en la línea del aprendizaje, también se encuentra la comunicación. Por ejemplo, en la CCFACC la comunicación de precios y características de los productos ofrecidos se hace en el portal virtual para que todos los asociados estén en contacto. Por otra parte, se detectan los boletines institucionales y los de novedades comerciales que sirven como herramienta de difusión de información.

En cuanto a la experiencia, en el caso de la CCFACC, cerca del $70 \%$ de las entidades ya formaban parte de otra central de compras (Primer Precio). Según el secretario de la FACC, "Primer Precio hoy es una experiencia que nosotros estamos capitalizando a través de la central de compras. Esa experiencia la tomamos como una excelente idea que trajo una solución a una problemática y, a su vez, nuestro portal la ha mejorado aún más". Por su parte, se podría decir que la Coop Italia está utilizando su experiencia de integración a nivel nacional para iniciarse en la integración internacional, al aliarse a Coopernic junto a cuatro redes de compras europeas.

Por otra parte, en relación a la claridad de derechos y obligaciones de los participantes, los dos modelos lo resuelven fácilmente al implementar contratos escritos para habilitar la participación de alguna cooperativa a la central. De esta forma, se evitan confusiones y cada uno sabe exactamente cuáles son sus derechos y obligaciones por formar parte del proyecto.

En el caso italiano los mecanismos de control descansan, básicamente, en los contratos escritos que firma cada uno de los asociados al decidir participar del proyecto. Por lo tanto, los mismos se comprometen a actuar de la forma indicada por la Coop en el convenio. Según el informe de la ANCECO (2016), la mayoría de las centrales de compras españolas no contaban con regímenes sancionadores 0 , en caso de tenerlos, no los aplicaban, justamente por el temor al abandono de la central.

En referencia al contexto, por ejemplo, a la CCFACC le está afectando la existencia de otras centrales de compra: "existe una experiencia en el centro-norte del país de supermercados mayoristas que hacen las veces de central de compras porque están ofreciendo líneas de productos muy variadas a precios competitivos con algunos otros servicios que tienen que ver con el marketing", de acuerdo a lo relatado por el presidente de la FACC. Este hecho genera que algunas cooperativas no estén tan interesadas en participar en la central cooperativa, debido a que encuentran el beneficio mediante otras redes. Además, esta central también se ve afectada por redes de distribución logística ya establecidas que las cooperativas difícilmente abandonan. 
Por otro lado, las políticas públicas en todos los casos van a afectar favorablemente o no a cualquier proyecto de tal envergadura. Por ejemplo, en Argentina las leyes de tributación implicarían una doble tributación si los pagos pasaran por medio de la central, por lo que el organismo lo resolvió vía la facturación directa proveedor-cooperativa de consumo. Al mismo tiempo, referido al caso italiano, Barberini (2009) afirma que las cooperativas están condicionadas por restricciones legales, financieras y administrativas que ponen limitaciones a su desempeño económico. Más aún: "En la realidad actual hay que decir, con toda claridad, que la contribución fiscal de las cooperativas, en su conjunto, puede considerarse equivalente, y en más de un caso superior, a la de las empresas que compiten con ellas" (Barberini, 2009: 180).

\section{Conclusiones}

Tras haber detectado los factores clave para un buen funcionamiento de una central de compras y haber realizado el análisis entre las dos unidades de análisis seleccionadas sobre cómo acciona cada una en relación a cada variable, se verifica que las dos centrales actúan proactivamente en relación a éstos y, de esta forma, logran una performance satisfactoria.

Es posible verificar que tanto la confianza como el compromiso son características que están más afianzadas en el caso de la Coop Italia, dado que son aspectos que se logran a través del tiempo y con la buena performance demostrada, lo que no quiere decir que no se dé en la CCFACC. Se debe tener en cuenta que es un proceso que se encuentra en sus fases iniciales y resulta difícil pretender confianza y compromiso hacia un proyecto poco conocido y experimentado.

Por otra parte, se comprueba que en ninguno de los dos casos se da la homogeneidad de tamaño; al contrario, se encuentran cooperativas de diferentes tamaños y dispersas geográficamente a nivel nacional. Sí se encuentra complementariedad y compatibilidad, dado que en ambas experiencias llegan a operar de forma satisfactoria terminando beneficiadas todas las partes.

Aunque no se verifica la homogeneidad en términos físicos, sí se comprueba una homogeneidad en cuanto a los principios y valores cooperativos compartidos, teniendo siempre como eje central al ser humano. Se encuentra que en ambos casos la razón fundamental de la central es el sexto principio cooperativo "cooperación entre cooperativas", apostando por una economía sostenible.

Asimismo, es interesante destacar que algunos de los resultados obtenidos no se habían registrado en la literatura. Por un lado, en lo referente a los factores clave, los aciertos fueron los siguientes: 
- Competitividad (surtido y precio): para el sector de retail específicamente, el surtido y los precios son clave para la competitividad. En realidad, en la literatura no se observa planteado como un factor clave, pero sí se verifica como un problema en las experiencias anteriores.

- Marketing homogéneo: la Coop Italia lo implementa y los resultados son exitosos sobre todo al dar una imagen de integridad entre los diferentes supermercados cooperativos. La CCFACC también comenzó a implementarlo de una manera más incipiente.

- Marca propia: se ha comprobado que la comercialización de la marca propia en una central de compras cooperativa es una herramienta fundamental tanto como una estrategia de diferenciación como de rentabilidad por contar con márgenes superiores. Tanto la Coop Italia como la CCFACC cuentan con marca propia y un alto porcentaje de las ventas pertenecen a ésta.

A su vez, también se destaca la clasificación de los factores clave estudiados. En este sentido, se propuso la siguiente agrupación según su naturaleza:

- Factores generadores de conductas actitudinales: aquellos que determinan la posición de las cooperativas asociadas ante el proyecto.

- Factores organizacionales: propios de la gestión, que pueden ir variando dependiendo del progreso del proyecto.

- Factores comerciales: relacionados a la actividad comercial, tendentes a lograr la eficiencia.

- Factores externos: variables relacionadas con el contexto.

Dicha clasificación fue útil para el ordenamiento de los factores, lo que permite una lectura más ágil de los resultados. Además, ayuda relacionar cada uno de ellos con las distintas fases del ciclo de vida de una central de compras cooperativa.

Por último, respondiendo a los interrogantes planteados en el inicio del estudio, es posible asegurar que las cooperativas están en condiciones de ser eficientes, teniendo en cuenta el caso de la Coop Italia que cuenta con una amplia experiencia de crecimiento y aún continúa en expansión. El caso de la CCFACC es incipiente, pero, según lo estudiado, se podría concluir en que está pensada y comenzando a funcionar en base a los factores claves. A su vez, ambos casos siempre actúan en el marco de sus principios y valores, apoyándose en éstos para construir su identidad cooperativa, su aspecto distintivo. 


\section{Referencias}

ACOSTA, M. \& VERBEKE, G. (2009): La cooperación como estrategia de desarrollo en redes asociativas, Pecvnia, 9, 1-25. DOI: http://dx.doi.org/10.18002/pec.v0i9.658.

ADOBOR, H. \& MCMULEN, R. (2014): "Strategic purchasing and supplier partnerships - The role or a third-party organization", Journal of Purchasing \& Supply Management, 20, 263-272. DOI: https://doi.org/10.1016/j.pursup.2014.05.003.

ALIANZA COOPERATIVA INTERNACIONAL: http://www.aciamericas.coop/ Última vez consultada: Octubre de 2018.

ALIANZA COOPERATIVA INTERNACIONAL (2013): Plan para una década cooperativa. http://www.aciamericas.coop.

ANCECO (2004): "Estudio realizado por UGAL. Las CCS europeas: factores para el éxito, estrategias clave, consolidación de posiciones", ANCECO Estudios, 3.

ANCECO (2016): "Presente y futuro de las CCS españolas", Revista Central, 4. Noviembre.

BACIC, M.J., DE SOUZA, F. \& AVEZEDO, M.C. (2008): "Formación de redes horizontales de pequeñas empresas como estrategia de obtención de ventajas competitivas: análisis de una experiencia", Revue Sciences de Gestión, 66.

BARBERINI, I. (2009): EI Vuelo del Abejorro: Cooperativismo, Ética y Desarrollo, Argentina, Intercoop Editora Cooperativa Limitada.

BASTIDAS-DELGADO, O. (2015): La Identidad Cooperativa, Editora Galixia C.A., Venezuela.

BRAZDA, J. \& SCHEDIWY, R. (2003): "Esbozo histórico de las cooperativas de consumo", CIRIECEspaña, Revista de Economía Pública, Social y Cooperativa, 44, 105-136.

CALDERWOOD, E. \& DAVIES, K. (2013): "Co-operatives in the retail sector: can one label fit all?", Journal of Co-operative Studies, 46, 16-31.

CASAL, J. \& MATEU, E. (2003): "Tipos de muestreo", Revista Epidem, Med. Prev., 1, 3-7.

CHARTERINA, A.M. (2012): "Sobre el principio de cooperación entre cooperativas en la actualidad", Boletín de la Asociación Internacional de Derecho Cooperativo, 46, 133-146.

DOI: http://dx.doi.org/10.18543/baidc-46-2012pp133-146.

DAVIES, K. \& BURT, S. (2007): "Consumer co-operatives and retail internationalization: Problems and prospects", International Journal of Retail and Distribution Management, 35(2), 156-177. DOI: https://doi.org/10.1108/09590550710728101. 
GAEDA, E. (2011): "Cooperativismo de consumo: la adaptación del modelo a las nuevas condiciones del mercado", Boletín de la Asociación Internacional de Derecho Cooperativo, 45, 191-196.

HERNÁNDEZ, R., FERNÁNDEZ, C. \& BAPTISTA, M. (2010): Metodología de la investigación, Quinta edición, México, McGraw-Hill.

HOFFMAN, W.H. \& SCHLOSSER, R. (2001): "Success factors of strategic alliances in small and medium-sized enterprises. An empirical survey", Long range planning, 34(3), 357-381. DOI: https://doi.org/10.1016/S0024-6301(01)00041-3.

LAGEMANN, L. (2004): Fatores que influenciam a performance de redes de pequenas e médias empresas, Tesis de posgrado en Administración, Universidade Federal do Rio Grande do Sul, Brasil.

MENZANI, T. \& ZAMAGNI, V. (2010): "Cooperative networks in the Italian economy", Enterprise and Society, 11(1), p. 98. DOI: https://doi.org/10.1017/S1467222700008570.

MIGLIAVACCA, E. (2016): La Coop nell'attuale contesto competitivo, http://www.aciamericas.coop/ivcumbre.

PORTER, M.E. (1980): Competitive strategy, New York, Free Press.

MONTES, V.L. \& RESSEL, A.B. (2003): "Presencia del Cooperativismo en Argentina", Revista UniRcoop, 1.

POUSA, R. (2006): Os Ciclos de Vida das Centrais de Negócios: Um Estudo no Setor Supermercadista no Estado de São Paulo, XIII SIMPEP-Simpósio de engenharia de produção. Bauru/SP, 6.

REGALADO, J.C.P., GONZÁLEZ, A.N. \& ECHEVERRÍA, L.R. (2017): "Formas de integración cooperativa y sus principales técnicas de realización. La intercooperación cooperativa/Forms of cooperative integration and its mains techniques of realization cooperative intercooperation", Estudios del Desarrollo Social: Cuba y América Latina, 5 (3), 85-99.

RYAN, G. \& BERNARD, H.R. (2003): "Techniques to identify themes", Field Methods, 15 (1), 85-109. DOI: https://doi.org/10.1177/1525822X02239569.

RUBIO, M.A. (2017): "Análisis crítico de la fiscalidad de las cooperativas de segundo grado como fórmula de integración", REVESCO, Revista de Estudios Cooperativos, 126, 118-132. DOI: https://dx.doi.org/10.5209/REVE.58393.

SANCHIS, J.R., CAMPOS, V. \& MOHEDANO, A. (2015): "Factores clave en la creación y desarrollo de cooperativas. Estudio empírico aplicado a la comunidad valenciana", REVESCO, Revista de Estudios Cooperativos, 119, 183-207.

DOI: http://dx.doi.org/10.5209/rev_REVE.2015.n119.49153.

SOUZA, M.G. (2004): Centrais de Negócios. Uma revolução no varejo e na distribuição. São Paulo, Edições Inteligentes. 
SUÁREZ, F., FASSIO, A. \& PASCUAL, L. (2004): Introducción a la metodología de la investigación, Ediciones Macchi, Buenos Aires.

TENCATI, A. \& ZSOLNAI, L. (2009): "The collaborative enterprise", Journal of Business Ethics, 85 (3), 367-376. DOI: 10.107/s10551-008-9775-3.

TORRES, F., MURGADO, E.M., SENISE, O., MOZAS, A. \& PARRAS, M. (2006): Proyecto Elaboración de un estudio dirigido a la creación de una central de compras y de servicios en el sector oleícola provincial, suscrito entre la Excma. Diputación provincial de Jaén y la Universidad de Jaén.

TRAVISAN, L. (2016): L'evoluzione digitale del retail e customer experience. Caso Coop Italia: Future Food District, Bachelor's Thesis, Univeritá Ca'Foscari Venezia.

VILLARREAL, O. \& LANDETA, J. (2010): "El estudio de casos como metodología de investigación científica en dirección y economía de la empresa. Una aplicación a la internacionalización", Investigaciones Europeas de Dirección y Economía de la Empresa, 16 (3), 31-52. DOI: https://doi.org/10.1016/S1135-2523(12)60033-1.

VUOTTO, M. (2013): Investigar sobre cooperativas, Publicación N¹9, Segunda Serie GIDECOOP UNS.

YIN, R.K. (2001): Estudio de Caso. San Pablo, Artmed. 
